\title{
Morphological, Indumentum and Chemical Characteristics and Analysis of the Volatile Components of the Flowers of Rhododendron ponticum L. subsp. ponticum (Ericaceae) of Turkish Origin $^{\S}$
}

\author{
Sevim Küçük ${ }^{\oplus 1^{*}}$, Mine Kürkçüoğlu ${ }^{\oplus 2}$ and Kemal Hüsnü Can Başer ${ }^{\oplus 3}$ \\ ${ }^{I}$ Department of Pharmaceutical Botany, Faculty of Pharmacy, Anadolu University, 26470, Eskisehir, Türkiye \\ ${ }^{2}$ Department of Pharmacognosy, Faculty of Pharmacy, Anadolu University, 26470 Eskişehir, Türkiye \\ ${ }^{3}$ Near East University, Faculty of Pharmacy, Department of Pharmacognosy, Near East Boulevard, \\ ZIP: 99138, Nicosia, Turkish Republic of Northern Cyprus
}

(Received November 6, 2017; Revised January 29, 2018; Accepted January 30, 2018)

\begin{abstract}
Morphological, chemical and indumentum characteristics of Rhododendron ponticum L. subsp. ponticum collected from Bartın province have been investigated. A detailed description of the species has been prepared and compared with that published in the Flora of Turkey of Davis in a tabular form. The taxonomic and morphological characteristics of the plant material have been described and illustrated by drawings. Indumentum characteristics were also investigated.Volatiles of the flowers were trapped by a dynamic headspace SPME setup and their hexane extract were analyzed by GC/MS. Main components of the headspace trapped volatiles of flowers were characterized as $\alpha$-pinene (44.5\%), $\beta$-pinene $(10.8 \%)$, linalool $(4 \%)$ and limonene $(3.3 \%)$ whereas main components in volatiles of the hexane extract were linalool (19.6\%), phenylethyl alcohol (19.1\%), myrtenol (10.1 $\%)$. citronellol (9.4\%) and phenylacetaldehyde (7.8\%).
\end{abstract}

Key words: Rhododendron ponticum subsp. ponticum, ericaceae; morphology; indumentum; chemistry, GC-MS HS-SPME. (C) 2018 ACG Publications. All rights reserved.

\section{Introduction}

More than 850 species of Rhododendron L. (Orman Gülü) are distributed in the Northern Hemisphere. The genus Rhododendron distributed in North Eastern and West Anatolia is represented by 9 species two being endemic and altogether 12 taxa, 4 hybrids and 1 form [1-5].

These are Rhododendron. luteum Sweet (Sarı çiçekli ormangülü), $R$. ungernii Trautv. (Ak Ormangülü), $R$. smirnowii Trautv. (Kızıl Kumar) and., $R$. caucasicum Pallas (Kafkas Ormangülü), $R$. ponticum L. subsp. ponticum (Mor Çiçekli Ormangülü), $R$. ponticum subsp. ponticum forma album, $R . \mathrm{x}$ sochadzeae Charadze \& Davlianidze, $R$. x rosifaciens R.Milne, $R$. x davisianum R.Milne, $R . \mathrm{x}$ filidactylis R.Milne [1-5].

Leaves and flowers of Rhododendron (especially $R$. luteum and $R$. ponticum) contain toxic compounds (andromedotoxin). Honey from the flowers of Rhododendron is locally known as 'deli bal,

\footnotetext{
*Corresponding author: E-Mail: salan@ anadolu.edu.tr; Phone:+90-545-2997214

${ }^{\S}$ Presented at the 10th International Symposium on the Chemistry of Natural Compounds (10th SCNC), 21-23 November 2013, Tashkent, Uzbekistan
} 
ac1 bal, tutar bal" (Mad honey). It has antihypertension activity and when consumed it inflicts neurotoxicity by causing light-headedness and hallucinations. Rhododendron species are also used as decorative plants [6-10]. When consumed, consciousness disorders similar to drunkenness are observed. Overdose is lethal. Leaves of $R$. luteum (Kumar leaf) contain tannin, essential oil, ericolin, arbutin and andromedol derivatives. Although used as diuretic and analgesic in rheumatic pains its infusion can be dangerous due to andromedol derivatives. A decoction of $R$. luteum and $R$. ponticum leaves is externally used to treat fungal foot infectious in Giresun province (Dereli, Çalca Eğriambar). R. luteum is also used as ornamental due to its showy flowers [6-10].

$R$. ponticum is an evergreen plant, growing up to $10 \mathrm{~m}$ in Northern and Eastern Anatolian mountains. This plant known as 'Mor çiçekli ormangülü, Alp gülü, Komar, Karaağu, Kara Kumar, Kumar'” [6-10].

The volatile profile of some Rhododendron species have been investigated. 1-Methyl-2-pyrrolidone was reported as main compound in the $\mathrm{CH}_{2} \mathrm{Cl}_{2}$ extract flowers of Rhododendron ponticum L. [11].

This report concerns morphological, chemical and indumentum characteristics of Rhododendron ponticum subsp. ponticum collected from Bartın province. A detailed description of the species has been prepared and compared with that published in the Flora of Turkey of Davis in a tabular form. The taxonomic and morphological characteristics of the plant material have been described and illustrated by drawings. Indumentum characteristics were also investigated. Volatiles of the flowers and their hexane extract were trapped by a dynamic Headspace Solid-Phase Microextraction setup and were analyzed by GC-MS (HS-SPME-GC-MS).

This is the first report on the morphological, indumentum characteristics and headspace volatiles and hexane extract of the flowers of this plant.

\section{Materials and Methods}

\subsection{Plant Material}

Aerial parts were collected in May 2011, A4 Bartın: Hasan kadı beldesi, Akbay köyü, 300 m. Voucher specimens are kept at the Herbarium of the Faculty of Pharmacy, Anadolu University in Eskişehir, Turkey (ESSE 14428).

\subsection{Morphological and Anatomical Studies}

The plant material was identified as $R$. ponticum subsp. ponticum using the Flora of Turkey and the East Aegean Islands (Stevens 1978). Herbarium specimens were used for description and detailed morphological drawings. A Leitz SM-LUX binocular microscope with drawing tube was used for anatomical drawings. A wild M5 A stereo microscope with drawing tube was utilized for morphological drawing.

\subsection{Headspace-Solid Phase Micro Extraction (HS-SPME) Analysis}

60 min sampling was carried out on live plant materials using a blue fibre PolydimethylsiloxaneDivinylbenzene (PDMS/DVB - 65 $\mu \mathrm{m}$ ) - Blue (supplied by Supelco Bellefonte, USA). The fiber was directly desorbed in GC/MS for $10 \mathrm{~min}$.

Headspace-SPME procedure for the extract: The volatiles were trapped by SPME. SPME fiber coated with PDMS/DVB - $65 \mu \mathrm{m}$ was used with a sampling time of $15 \mathrm{~min}-50^{\circ} \mathrm{C}$. Thermal desorption at $250^{\circ} \mathrm{C}$ for $10 \mathrm{~min}$.

\subsection{GC/MS Conditions}

The GC/MS analysis was carried out with an Agilent 5975 GC-MSD system. Innowax FSC column (60m $\times 0.25 \mathrm{~mm}, 0.25 \mu \mathrm{m}$ film thickness) was used with helium as carrier gas $(0.8 \mathrm{~mL} / \mathrm{min}$.). GC oven temperature was kept at $60{ }^{\circ} \mathrm{C}$ for $10 \mathrm{~min}$ and programmed to $220{ }^{\circ} \mathrm{C}$ at a rate of $4{ }^{\circ} \mathrm{C} / \mathrm{min}$, and kept constant at $220{ }^{\circ} \mathrm{C}$ for $10 \mathrm{~min}$ and then programmed to $240{ }^{\circ} \mathrm{C}$ at a rate of $1{ }^{\circ} \mathrm{C} / \mathrm{min}$., at splitless mode. The injector temperature was at $250{ }^{\circ} \mathrm{C}$. MS were taken at $70 \mathrm{eV}$. Mass range was from m/z 35 to 450 . 
The components of essential oils were characterized by comparison of their mass spectra with those in the Baser Library of Essential Oil Constituents, Wiley GC/MS Library, Adams Library, MassFinder Library and confirmed by comparison of their retention indices. Alkanes were used as reference points in the calculation of relative retention indices (RRI). The results of analysis are shown in Table 2.

\section{Results and Discussion}

\subsection{Morphological Observations}

Rhododendron ponticum subsp. ponticum (Figure 1) is a large shrub, to $10 \mathrm{~m}$ Stem with variable indumentum when young, but not tomentose, glabrous-glandular; terminal bud ca.1.5$2 \mathrm{~cm}$. Petiole 1-1.7 cm, glabrous-glandular; lamina eliptic-obovate, 6-12.5 $\times 2-4.5 \mathrm{~cm}$, base acute, margin repant-integer, obtuse-acute at apex, coriaceous, glabrous-glandular hairs, fine venation usually flat above.

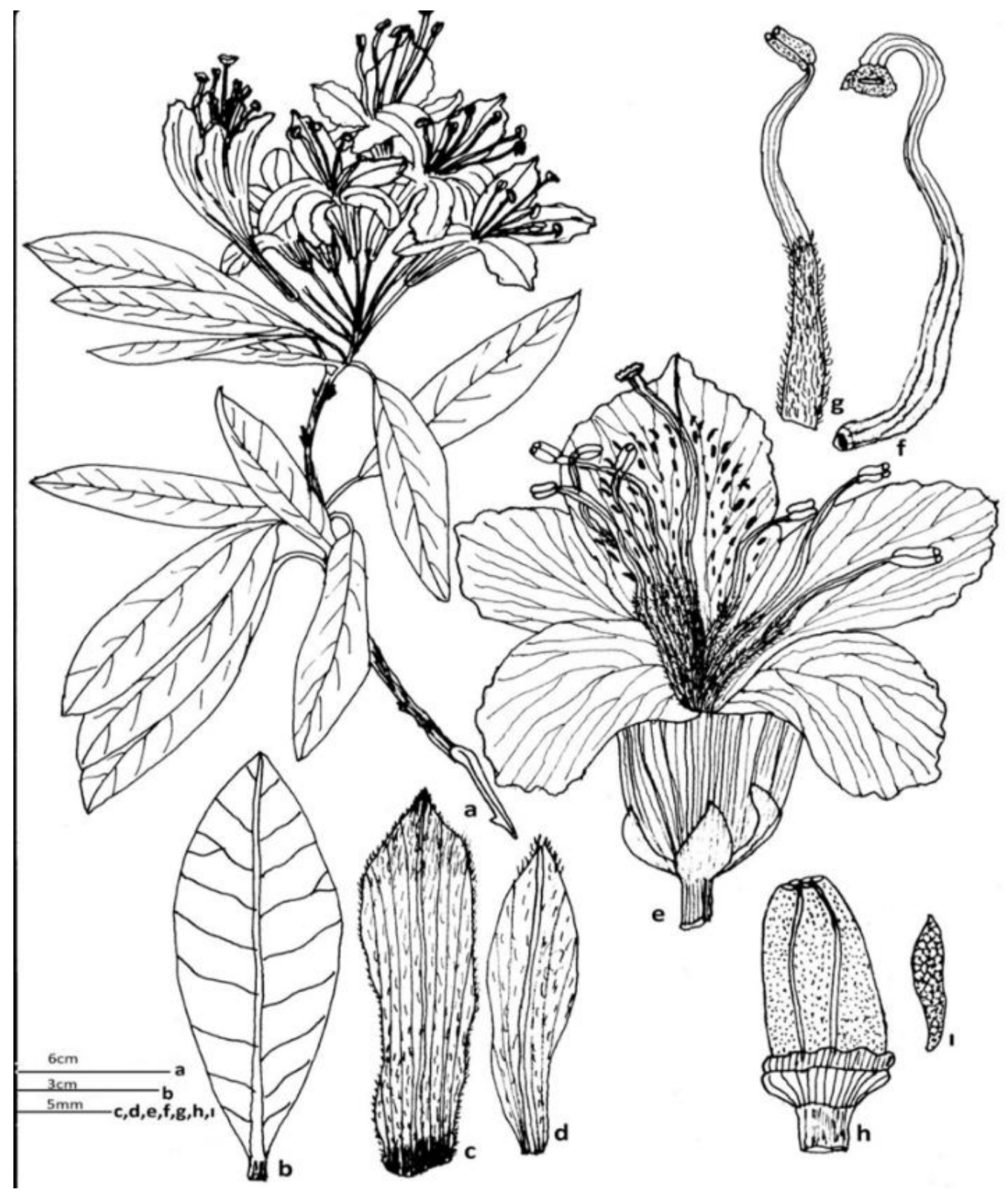

Figure 1. Rhododendron ponticum subsp. ponticum. a-plant; b-leaf; c-- bracts; d-bracteols; e-flower; f-- pistil; g-- stamen; h-- capsule; 1-- nutlets. 
Inflorescence terminal, racemous, 7-25 flowered, axis $5-7.5 \mathrm{~cm}$, peduncle $1.5-8 \mathrm{~cm}$; pedicels $2-3 \mathrm{~cm}$, glabrous glandular hairs, bracts $6-10 \times 2-3.5 \mathrm{~cm}$, ovate-linear, decidous and bracteols 1-2.5 cm, pubescent-eglandular. Calyx 5 lobes, ovate-linear, lobes 0.6-4 mm, glabrous-glandular. Corolla 2.8-3.6 cm, purplish-pink, upper with yellow spots, campanulate, 2-8x3-6 cm, 1-2 cm across, 5 lobes, acute-rounded, lobes $1.5-2.5 \mathrm{~cm}$, usually glabrous outside, pubescent towards base inside, tube 1.2-1.6 cm, Stamens 6-10, 2.5-4 cm. Anther dorsifixed, pink,2.3-3 mm. Filament 2.2-3.7 cm, pink, base pubescent. Ovary 4-5x1.5-2 cm, cylindric, 5locular, glabrous-eglandular; style 3-4 cm, glabrous-glandular; stigma capitate, pink -pale brown. Capsule 1.5-3 cm, septicidal capsule; seeds ca. $2.5 \mathrm{~mm}$ (Figure 1). Flowering period: (3-)5-6(-8). Habitat: Usually in Fagus orientalis forest, rarely in other types of forest or above tree line. Altitude: (s.1.-)150-1800 (-2100) m. Distribution in Turkey: N. Turkey. E. Balkans, Caucasus, Georgia, Lebanon. Euxine element [3].

Table 1. Morpho-metrical characters of Rhododendron ponticum subsp. ponticum (Figure 1)

\begin{tabular}{|c|c|c|c|}
\hline \multicolumn{2}{|c|}{ Morpho-metrical characters } & Flora of Turkey & $\begin{array}{l}\text { The findings of this } \\
\text { study }\end{array}$ \\
\hline \multicolumn{2}{|c|}{ Indumentum of stem } & variable when young, but not tomentose & Glabrous, glandular \\
\hline \multirow[t]{4}{*}{ Petiole } & indumentum & -- & Glabrous, glandular \\
\hline & size & $(6-) 10-17 \times(2.8-) 3.5-4.5 \mathrm{~cm}$ & $6-12.5 \times 2-4.5 \mathrm{~cm}$ \\
\hline & base & -- & Acute \\
\hline & margin & -- & Repand-integer \\
\hline \multirow[t]{2}{*}{ Lamina } & apex & -- & Obtuse-acute \\
\hline & indumentum & $\begin{array}{l}\text { Coriaceous, initially tomentose below but } \\
\text { soon glabrescent, glandular hairs persistent }\end{array}$ & Glabrous, glandular \\
\hline \multirow{3}{*}{$\begin{array}{l}\text { Inflorescenc } \\
\text { es }\end{array}$} & Number of flowers & $5-20$ & $7-25$ \\
\hline & shape & Terminal & Terminal rasemous \\
\hline & axis & $1.3-7 \mathrm{~cm}$ & $5-7.5 \mathrm{~cm}$ \\
\hline \multirow[t]{2}{*}{ Peduncle } & size & $1.3-7 \mathrm{~cm}$ & $1.5-8 \mathrm{~cm}$ \\
\hline & size & $2-2.5 \mathrm{~cm}$ & $2-3 \mathrm{~cm}$ \\
\hline \multirow[t]{2}{*}{ Pedicel } & shape & $\begin{array}{l}\text { Usually glabrous, sometimes with glandular } \\
\text { hairs or pubescent }\end{array}$ & Glabrous, glandular \\
\hline & shape & Ovate-linear & Ovate-linear, deciduous \\
\hline \multirow[t]{3}{*}{ Bracts } & size & $2.5-3.5 \mathrm{~cm}$ & $6-10 \times 2-3.5 \mathrm{~cm}$ \\
\hline & indumentum & -- & Pubescent, eglandular \\
\hline & Shape & -- & $\begin{array}{l}\text { Ovate -linear, } \\
\text { deciduous }\end{array}$ \\
\hline \multirow[t]{4}{*}{ Bracteols } & Size & $2.5-3.5 \mathrm{~cm}$ & $1-2.5 \mathrm{~cm}$ \\
\hline & İndumentum & -- & Pubescent, eglandular \\
\hline & Shape of lobes & -- & Ovate-linear \\
\hline & Size of lobes & $0.6-1(-3) \mathrm{mm}$ & $0.6-4 \mathrm{~mm}$ \\
\hline \multirow{2}{*}{ Calyx } & Number of lobes & -- & 5 \\
\hline & İndumentum & & Glabrous, glandular \\
\hline
\end{tabular}




\begin{tabular}{|c|c|c|c|}
\hline & Table 1 Continued.. & & \\
\hline \multirow{9}{*}{ Corolla } & colour & Purplish-pink,upper with yellow spots & Same \\
\hline & shape & Campanulate & Campanulate \\
\hline & size & -- & $2.8-3.6 \mathrm{~cm}$ \\
\hline & Shape of lobes & Acute-rounded & Acute-rounded \\
\hline & Number of lobes & -- & 5 \\
\hline & Size of lobes & $1.5-2.5 \mathrm{~cm}$ & $1.5-2.5 \mathrm{~cm}$ \\
\hline & Across of lobes & $1.5-2.5 \mathrm{~cm}$ & $1-2 \mathrm{~cm}$ \\
\hline & Size of tube & $1.5-2.5 \mathrm{~cm}$ & $1.2-1.6 \mathrm{~cm}$ \\
\hline & Indumentum & $\begin{array}{l}\text { Usually glabrous outside, pubescent towards } \\
\text { base, inside pubescent }\end{array}$ & Same \\
\hline \multirow{3}{*}{ Stamen } & number & 10 & $6-10$ \\
\hline & size & -- & $2.5-4 \mathrm{~cm}$ \\
\hline & shape & -- & Dorsifixed \\
\hline \multirow[t]{3}{*}{ Anther } & colour & -- & Pink \\
\hline & size & -- & $2.3-3 \mathrm{~mm}$ \\
\hline & size & -- & $2.2-3.7 \mathrm{~cm}$ \\
\hline \multirow{3}{*}{ Filament } & colour & -- & Pink \\
\hline & Table 1 Continueed.. & & \\
\hline & indumentum & -- & Base pubescent \\
\hline \multirow{3}{*}{ Ovary } & size & -- & $4-5 \times 1.5-2 \mathrm{~cm}$ \\
\hline & shape & 5-locular & Cylindrical, 5-locular \\
\hline & indumentum & Glabrous & Glabrous, eglandular \\
\hline \multirow{2}{*}{ Style } & size & $3-4 \mathrm{~cm}$ & $3-4.5 \mathrm{~cm}$ \\
\hline & indumentum & Glabrous & Glabrous, glandular \\
\hline \multirow{2}{*}{ Stigma } & shape & Capitate & Same \\
\hline & colour & -- & Pink -Pale brown \\
\hline \multirow{2}{*}{ Capsule } & shape & Septicidal capsule & Same \\
\hline & size & $1.6-2.8 \mathrm{~cm}$ & $1.5-3 \mathrm{~cm}$ \\
\hline Seeds & size & c. $2 \mathrm{~mm}$ & $2.5 \mathrm{~mm}$ \\
\hline
\end{tabular}

Leaves elliptic-obovate, glabrous-glandular hairs on surfaces. Flowers are pink- purple. Flowers are usually with 5 fragments. Flowering period, usually in summer months [3].

To the best of our knowledge, there is no morphological study on $R$. ponticum subsp. ponticum. Morphological and morphometric descriptions are compared with those in the Flora of Turkey as shown in Table 1 and Figure 1. Most of our findings were in agreement with those features published in the Flora of Turkey. However, lower limits of leaf, pedicel, style and fruit dimensions were found to be higher in our findings. This is possibly due to the number of specimens investigated and to the ecological reasons. Here, we also report for the first time the base, margin, shape of lamina, type of inflorescence, calyx tooth shape and number, corolla length, shape and number of lobes, stamen length, shape colour and length of anther, colour and length of filament, ovary size and shape, style colour, stigma shape and colour, seed colour characteristics [3] . 


\subsection{Indumentum}

Indumentum characteristics of stem, leaf, pedicel calyx, corolla, ovary and style were investigated by anatomical studies in comparison with those given in the Flora of Turkey in Table 1. Covering trichomes are simple with 1-2 cells; glandular hairs are emergence. Indumentum characters deviated from those reported by Davis [3]. In addition to those characters published in Flora of Turkey, we also observed glabrous-glandular on the stem and glabrous-glandular on the pedicel. Covering trichomes on the leaves are glabrous-glandular. Bracts, bracteols and ovary are glabrous-eglandular. Calyx and style are glabrous-glandular (Figure 2).

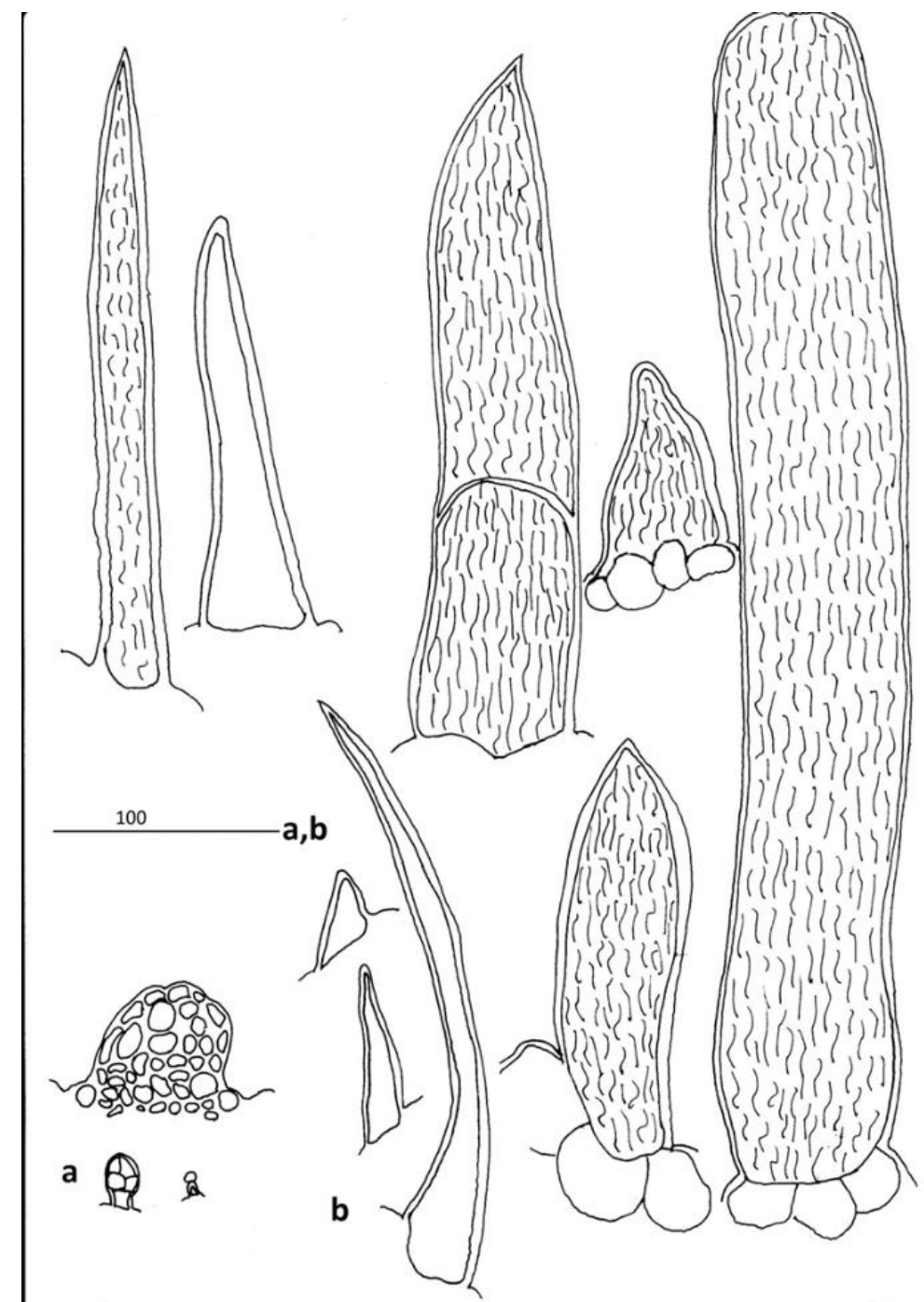

Figure 2. In light microscope of hairs of Rhododendron ponticum subsp ponticum. a- glandular hairs (emergence); b- covering hairs

\subsection{Composition}

Essential oils of some Rhododendron species have been investigated. Mostly terpenes such as $\alpha$ humulene, caryophyllene, limonene, $\alpha$ - or -pinene comprise the main constituents of the essential oils. A study reported mostly non-terpenic hydrocarbons, alcohols, esters and ketones as the major components [11]. The main poisonous constituents of Rhododendron plants, grayanotoxins (andromedotoxins), were not detected in any of the extracts. These diterpenes are unstable on heating and have low vapor pressure, hence they require derivatization (TMS) before the GC analysis Andromedotoxin has been isolated from the waste material in the production of Rhododendron essential oils [11]. 
Table 2. Composition of the flower volatiles of R. ponticum subsp. ponticum

\begin{tabular}{|c|c|c|c|c|c|}
\hline $\mathbf{R R I}^{\mathbf{a}}$ & $\mathbf{R R I}^{\mathbf{b}}$ & Compounds & $\begin{array}{c}\text { A } \\
\% * \\
\end{array}$ & $\begin{array}{l}\text { B } \\
\% \\
\end{array}$ & ID $^{* *}$ \\
\hline 1032 & $\begin{array}{c}1025^{\mathrm{c}, \mathrm{d}} \\
1032^{\mathrm{g}}\end{array}$ & $\alpha$-Pinene & 44.5 & 1.3 & RRI, MS \\
\hline $\begin{array}{l}1118 \\
1203\end{array}$ & $\begin{array}{l}1117^{\mathrm{c}, \mathrm{d}} \\
1212^{\mathrm{c,d}}\end{array}$ & $\begin{array}{l}\beta \text {-Pinene } \\
\text { Limonene }\end{array}$ & $\begin{array}{c}10.8 \\
3.3\end{array}$ & - & $\begin{array}{l}\text { RRI, MS } \\
\text { RRI, MS }\end{array}$ \\
\hline 1205 & & 3-Hexanol & - & 2.7 & RRI, MS \\
\hline 1213 & $\begin{array}{c}1013-1039^{\mathrm{f}} \\
1213^{\mathrm{n}}\end{array}$ & 1,8-Cineole & 0.8 & - & RRI, MS \\
\hline 1223 & & 2-Hexanol & - & 3.5 & MS \\
\hline 1266 & $1249^{c}$ & (E)- $\beta$-Ocimene & 1.3 & - & MS \\
\hline 1267 & & 3-Octanone & 1.4 & - & RRI, MS \\
\hline 1280 & $\begin{array}{l}1268^{\mathrm{e}} \\
1282^{\mathrm{d}}\end{array}$ & $p$-Cymene & 1.8 & - & RRI, MS \\
\hline 1360 & $1360^{\mathrm{n}, \mathrm{t}}$ & 1-Hexanol & 0.8 & 0.5 & RRI, MS \\
\hline 1400 & $1400^{\mathrm{n}}$ & Tetradecane & 1.1 & 0.3 & RRI, MS \\
\hline 1418 & & (Z)-2-Hexen-1-ol & - & 0.1 & RRI, MS \\
\hline 1450 & $1450^{\mathrm{c}}$ & $\begin{array}{l}\text { trans-Linalool oxide } \\
\text { (Furanoid) }\end{array}$ & - & 0.6 & MS \\
\hline 1452 & $1452^{\mathrm{h}}$ & $p$-Cymenene & 0.6 & - & MS \\
\hline 1453 & $1444-1452^{d}$ & 1-Octen-3-ol & 2.9 & 6.5 & RRI, MS \\
\hline 1466 & $\begin{array}{l}1466- \\
1480^{\mathrm{d}}\end{array}$ & $\alpha$-Cubebene & 0.2 & - & MS \\
\hline 1478 & $1445-1448^{c}$ & $\begin{array}{l}\text { cis-Linalool oxide } \\
\text { (Furanoid) }\end{array}$ & 0.3 & 1.7 & MS \\
\hline 1496 & $1496^{\mathrm{s}}$ & 2-Ethyl-1-hexanol & 1.1 & - & MS \\
\hline 1497 & $1488^{\mathrm{d}}$ & $\alpha$-Copaene & 1.4 & 0.5 & MS \\
\hline 1532 & $1515-1532^{d}$ & Camphor & 1.4 & - & RRI, MS \\
\hline 1535 & $\begin{array}{l}1523^{\mathrm{d}} \\
1535^{\mathrm{k}}\end{array}$ & $\beta$-Bourbonene & - & 0.5 & RRI, MS \\
\hline 1541 & $1541^{\mathrm{n}, \mathrm{s}}$ & Benzaldehyde & - & 1.0 & MS \\
\hline 1541 & & Isolongifolene & 0.8 & - & RRI, MS \\
\hline 1548 & $1548^{t}$ & (E)-2-Nonenal & - & 0.2 & MS \\
\hline 1553 & $1538^{\mathrm{c}}-1553^{\mathrm{c}, \mathrm{g}}$ & Linalool & 4.0 & 19.6 & RRI, MS \\
\hline 1562 & $1562^{t}$ & Octanol & - & 0.2 & RRI, MS \\
\hline 1583 & $1583^{r}$ & Longifolene & 1.7 & 0.1 & MS \\
\hline 1589 & $1576^{\mathrm{d}}$ & $\beta$-Ylangene & - & 0.4 & MS \\
\hline 1612 & $\begin{array}{l}1608- \\
1612^{c}\end{array}$ & $\beta$-Caryophyllene & 1.3 & 0.5 & RRI, MS \\
\hline 1621 & & 2-Octen-1-ol & - & 0.6 & MS \\
\hline 1641 & $1641^{\mathrm{t}}$ & Methyl benzoate & - & 0.3 & MS \\
\hline
\end{tabular}




\begin{tabular}{|c|c|c|c|c|c|}
\hline \multicolumn{6}{|c|}{ Table 2 Continued.. } \\
\hline 1648 & $1648^{k}$ & Myrtenal & 1.0 & 1.6 & MS \\
\hline 1658 & $1663^{\mathrm{s}}$ & Phenyl acetaldehyde & - & 7.8 & MS \\
\hline 1704 & $1689^{d}$ & $\gamma$-Muurolene & - & 0.3 & MS \\
\hline 1706 & $1695^{\mathrm{c}, \mathrm{e}}-1698^{\mathrm{e}}$ & $\alpha$-Terpineol & 1.6 & 2.2 & RRI, MS \\
\hline 1719 & $\begin{array}{c}1706^{\mathrm{c}, \mathrm{k}, \mathrm{g}} \\
1700-1701^{\mathrm{e}}\end{array}$ & Borneol & & & \\
\hline & $1720-1725^{\mathrm{d}}$ & Dent & 0.5 & 0.3 & RRI, MS \\
\hline 1725 & $1720-1725^{4}$ & Verbenone & 0.7 & - & RRI, MS \\
\hline 1726 & $\begin{array}{c}1711^{\mathrm{h}} \\
1722-1726^{\mathrm{c}}\end{array}$ & Germacrene D & - & 0.6 & MS \\
\hline 1733 & $1740^{\mathrm{m}}$ & Valencene & 0.5 & - & RRI, MS \\
\hline 1740 & $1723^{d}$ & $\alpha$-Muurolene & 0.2 & - & MS \\
\hline 1758 & $1758 n$ & $(E, E)-\alpha$-Farnesene & 0.7 & - & MS \\
\hline 1772 & $\begin{array}{l}1763^{\mathrm{f}, \mathrm{g}} \\
1772 \mathrm{n}\end{array}$ & Citronellol & 0.7 & 9.4 & RRI, MS \\
\hline 1773 & $\begin{array}{c}1755^{\mathrm{d}} \\
1750-1752^{\mathrm{e}}\end{array}$ & $\delta$-Cadinene & 0.5 & - & MS \\
\hline 1776 & $\begin{array}{c}1763^{\mathrm{d}} \\
1748-1749^{\mathrm{e}}\end{array}$ & $\gamma$-Cadinene & 0.4 & - & MS \\
\hline 1802 & $1790-1804^{\mathrm{d}, \mathrm{h}}$ & Myrtenol & 2.1 & 10.1 & MS \\
\hline 1849 & $1849-1927^{\mathrm{d}}$ & Calamenene & 0.3 & - & MS \\
\hline 1857 & $1839-1857^{\mathrm{d}, \mathrm{g}}$ & Geraniol & - & 1.5 & RRI, MS \\
\hline 1896 & $1896^{\mathrm{s}}$ & Benzyl alcohol & - & 1.8 & MS \\
\hline 1937 & $1937^{\mathrm{g}}$ & $\begin{array}{l}\text { Phenylethyl } \\
\text { alcohol }\end{array}$ & 0.5 & 19.1 & MS \\
\hline 2045 & $1988^{p}$ & Isopropyl myristate & 0.3 & - & MS \\
\hline
\end{tabular}

A: Headspace volatiles of the fresh flowers; B: Headspace volatiles of the hexane extract; RRI ${ }^{\mathrm{a}}$ : RRI Relative retention indices experimentally calculated against $n$-alkanes; RRI $^{\mathrm{b}}$ : RRI from literature (c [13]; d [14]; e [15]; f [16]; g [17]; h [18]; k [19]; m [20]; $\mathrm{n}$ [21]; p [22]; r [23]; s [24]; t [25]) for polar column values; *\% calculated from FID data; **ID: Identification Method; Identification method based on the relative retention indices (RRI) of authentic compounds on a HP Innowax column; MS, identified on the basis of computer matching of the mass spectra with those of the in-house Baser Library of Essential Oil Constituents, Adams [26], MassFinder [27] and Wiley [28] libraries.

In previous studies, headspace volatiles were reported from flowers of $R$. luteum. Main odour components were found as $\beta$-caryophyllene (34.0\%), methyl benzoate $(11.7 \%),(\mathrm{E})$ - $\beta$-ocimene $(10.4 \%)$ and $\alpha$-pinene $(10.0 \%)$ [12].

Headspace volatiles of fresh flowers of Rhododendron ponticum and the hexane extract were analyzed by gas chromatography/mass spectrometry. The volatiles were trapped by SPME in a dynamic headspace set up. A blue -Polydimethylsiloxane/Divinylbenzene (PDMS / DVB fibre was used.

Thirty -four and thirty-one volatile compounds were identified in both of the fresh flowers and hexane extract representing $91.5 \%$ and $95.8 \%$ of the total volatiles respectively.

Volatiles of $R$. ponticum subsp. ponticum were trapped by blue SPME fibre over a period of 1.5 hours. Main components were identified as $\alpha$-pinene $(44.5 \%)$, $\beta$-pinene $(10.8 \%)$, linalool $(4 \%)$ and limonene $(3.3 \%)$.

Volatiles of the hexane extract of $R$. ponticum were trapped on an HS-SPME Blue fibre. Main components were identified as linalool $(19.6 \%)$, phenylethyl alcohol $(19.1 \%)$, myrtenol $(10.1 \%)$, citronellol $(9.4 \%)$ and phenylacetaldehyde $(7.8 \%)$. 


\section{Acknowledgments}

The authors are grateful to Ali Ünsal Keskiner for collecting the plant materials.

\section{ORCID}

Sevim Küçük: 0000-0002-3594-0364

Mine Kürkçüoğlu: 0000-0002-9375-0294

Kemal Hüsnü Can Başer: 0000-0003-2710-0231

\section{References}

[1] M. Avcı (2004). Ormangülleri (Rhododendron L.) ve Türkiye'deki doğal yayılışları, İstanbul Üniversitesi, Edebiyat Fak. Coğrafya Bölümü, Coğrafya Dergisi, 12, 13-29.

[2] A. Güner, N. Özhatay, T. Ekim and K.H.C. Başer (2000). Flora of Turkey and East Aegean Islands, Edinburgh Univ. Press, 11:181-183, 322-339.

[3] P. F. Stevens (1978). Rhododendron L., Flora of Turkey and East Aegean Islands (Ed. PH. Davis), Edinburgh Univ. Press., 6, 91-94.

[4] S. Terzioğlu, N. Merey and R. Anşin (2001). A Study on Turkish Rhododendron L. (Ericaceae), Turk. J. Agric. Forest. 25, 311-317.

[5] A. Güner, S. Aslan, T. Ekim, M. Vural and M.T. Babaç (eds.) (2012). Türkiye Bitkileri Listesi (Damarlı Bitkiler). Nezahat Gökyiğit Botanik Bahçesi ve Flora Araştırmaları Derneği Yayını, İstanbul, 850-70.

[6] R. Acartürk (1997). Şifalı Bitkiler Flora ve Sağlığımız, Orman Genel Müdürlüğü Mensupları Yardımlaşma Vakfi, Yay. No:1,.88.

[7] A. Baytop (1991). Farmasötik Botanik Ders Kitabı, İst. Üniv. Eczacılık Fakültesi Yay. No:58, 224.

[8] T. Baytop (1984).Türkiye'de Bitkiler ile Tedavi, İst. Üniv. Yayın No: 3255 Ecz. Fak. Yay.No:40, $161,275$.

[9] E. Tuzlacı (2006). Şifa Niyetine, Türkiye'nin Bitkisel Halk İlaçları, Alfa Yayınları 1702, 283-284.

[10] N. Zeybek and U. Zeybek (1994). Farmasötik Botanik. Ege Üniversitesi Eczacılık Fakültesi Yay. No:2, 221-222.

[11] D. Taşdemir, B. Demirci, F. Demirci, A. Dönmez, K.H.C. Başer and Ruedi P, (2003). Analysis of the volatile components of five Turkish Rhododendron species by headspace solid-phase microextraction and GC-MS (HS-SPME-GC-MS), Z. Naturforsch. 58c, 797-803.

[12] S. Alan, M. Kürkçüoğlu, F. Göger and K.H.C. Baser, (2010). Morphological, chemical and indumentum characteristics of Rhododendron luteum sweet (Ericaceae), Pak. J. Bot., 42(6), 3729-3737.

[13] N. Kucukboyaci, B. Demirci, N. Adiguzel, B. Bani and K. H. C. Baser (2015). Volatile compounds from the aerial part and fruits of Grammosciadium pterocarpum Boiss. growing in Turkey, J. Essent. Oil Res. 27, 177-181.

[14] H. E. Temel, B. Demirci, F. Demirci, F. Celep, A. Kahraman, M. Dogan and K. H. C Baser (2016). Chemical characterization and anticholinesterase effects of essential oils derived from Salvia species, $J$. Essent. Oil Res. 28, 322-331.

[15] L. Solis-Quispe, C. Tomaylla-Cruz, Y. Callo-Choquelvica, A. Solís-Quispe, I. Rodeiro, I. Hernández, M. D. Fernández and J. A. Pino (2016). Chemical composition, antioxidant and antiproliferative activities of essential oil from Schinus areira L. and Minthostachys spicata (Benth.) Epl. grown in Cuzco, Peru, J. Essent. Oil Res. 28, 234-240.

[16] V. I. Babushok, P. J. Linstrom and I. G. Zenkevich (2011). Retention indices for frequently reported compounds of plant essential oils, J. Phys. Chem. Ref. Data 40, 043101-47.

[17] F. S. Senol, I. Erdogan Orhan, M. Kurkcuoglu, M. T. H. Khan, A., B. Sener and K. H. C. Baser (2013). A mechanistic investigation on anticholinesterase and antioxidant effects of rose (Rosa damascena Mill.), Food Res. Int. 53, 502-509.

[18] H. G. Agalar, M. Kurkcuoglu, A. Duran, O. Cetin and K. H. C. Baser (2015). Volatile compounds of Peucedanum chryseum (Boiss. et Heldr.) Chamberlain fruits, Nat. Vol. \& Essent. Oils (NVEO), 2(4), 4-10.

[19] N. Tan, D. Stana, B. Sen, E. Tan, H. B. Altan, B. Demirci and M. Uzun (2016). Antimycobacterial and antifungal activities of selected four Salvia species, Rec. Nat. Prod. 10, 593-603.

[20] S. Kucuk, M. Kurkcuoglu; Y. B. Köse and K. H. C. Baser (2015). Chemical Characterisation of the essential oil of Hypericum aviculariifolium Jaub. \& Spach subsp. depilatum (Freyn \& Bornm.) Robson var. bourgaei (Boiss.) Robson from Turkey, Nat. Vol. \& Essent. Oils (NVEO), 2 (2), 52-56.

[21] M. Kurkcuoglu, A. Abdel-Megeed, and K.H.C. Baser (2013). The composition of Taif rose oil, J. Essent. Oil Res., 25 (5), 364-367. 
[22] I. A. Schepetkin, S. V. Kushnarenko, G. Ozek, L. N. Kirpotina, G. A. Utegenova, Y. A. Kotukhov, A. N. Danilova, T. Ozek, K. H.C. Baser and M. T. Quinn (2015). Inhibition of human neutrophil eesponses by the essential oil of Artemisia kotuchovii and its constituents, J. Agric. Food Chem., 63, 4999-5007.

[23] O. Ustun, F. S. Senol, M. Kurkcuoglu , I. Orhan, M. Kartal and K.H.C. Baser (2012). Investigation on chemical composition, anticholinesterase and antioxidant activities of extracts and essential oils of Turkish Pinus species and pycnogenol, Ind. Crop. Prod. 38, 115-123.

[24] K. Polatoglu, B. Demirci, I. Calis and K.H.C. Baser (2017). Difference in volatile composition of Chenopodium murale from two different locations of Cyprus, Rec. Nat. Prod., 11 (1), 88-91.

[25] D. O. Moronkola, I. A. Ogunwande, K.H.C. Baser, T. Ozek and G. Ozek (2009). Essential oil composition of Gmelina arborea Roxb.,Verbenaceae, from Nigeria, J. Essent. Oil Res., 21 (3), 264-266.

[26] R. P. Adams (2007). Identification of Essential Oil Components by Gas Chromatography/Mass Spectrometry. Allured Publ. Corp, Carol Stream, IL.

[27] D. H. Hochmuth (2008). MassFinder-4, Hochmuth Scientific Consulting, Hamburg, Germany.

[28] F. W. McLafferty, D.B. Stauffer (1989). The Wiley/NBS Registry of Mass Spectral Data, J. Wiley and Sons: New York.

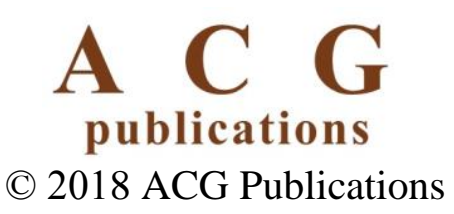

